\title{
SRAssembler: Selective Recursive local Assembly of homologous genomic regions
}

\author{
Thomas W. McCarthy ${ }^{1}$, Hsien-chao Chou ${ }^{2}$ and Volker P. Brendel ${ }^{1,3^{*}}$ (1)
}

\begin{abstract}
Background: The falling cost of next-generation sequencing technology has allowed deep sequencing across related species and of individuals within species. Whole genome assemblies from these data remain high time- and resource-consuming computational tasks, particularly if best solutions are sought using different assembly strategies and parameter sets. However, in many cases, the underlying research questions are not genome-wide but rather target specific genes or sets of genes. We describe a novel assembly tool, SRAssembler, that efficiently assembles only contigs containing potential homologs of a gene or protein query, thus enabling gene-specific genome studies over large numbers of short read samples.

Results: We demonstrate the functionality of SRAssembler with examples largely drawn from plant genomics. The workflow implements a recursive strategy by which relevant reads are successively pulled from the input sets based on overlapping significant matches, resulting in virtual chromosome walking. The typical workflow behavior is illustrated with assembly of simulated reads. Applications to real data show that SRAssembler produces homologous contigs of equivalent quality to whole genome assemblies. Settings can be chosen to not only assemble presumed orthologs but also paralogous gene loci in distinct contigs. A key application is assembly of the same locus in many individuals from population genome data, which provides assessment of structural variation beyond what can be inferred from read mapping to a reference genome alone. SRAssembler can be used on modest computing resources or used in parallel on high performance computing clusters (most easily by invoking a dedicated Singularity image).

Conclusions: SRAssembler offers an efficient tool to complement whole genome assembly software. It can be used to solve gene-specific research questions based on large genomic read samples from multiple sources and would be an expedient choice when whole genome assembly from the reads is either not feasible, too costly, or unnecessary. The program can also aid decision making on the depth of sequencing in an ongoing novel genome sequencing project or with respect to ultimate whole genome assembly strategies.
\end{abstract}

Keywords: Local assembly, NGS assembly, Genomics, Homolog

\section{Background}

Advances in next-generation sequencing (NGS) approaches have dramatically changed access to genome data, not only with respect to reference sequencing for many species, but increasingly for population studies of genomic variation (e.g., [1-3]. Applications of NGS include the creation of detailed maps of genetic variation [4-6], DNA methylation $[7,8]$, and transcription factor binding sites $[9,10]$.

\footnotetext{
*Correspondence: vbrendel@indiana.edu

${ }^{1}$ Department of Biology, Indiana University, Bloomington 47405, Indiana USA ${ }^{3}$ Department of Computer Science, Indiana University, Bloomington 47405, Indiana, USA

Full list of author information is available at the end of the article
}

Because NGS relies on extensive sequence coverage with small reads, accurate assembly of the reads into large contigs, scaffolds, and pseudochromosomes is an intrinsic part of the approach, and many NGS assembly tools have been developed for this purpose. Based on de Bruijn graphs [11], programs like Velvet [12], ABySS [13], ALLPATH [14], and SOAPdenovo2 [15] have been shown to effectively handle millions of short reads. Currently, research on genome assembly focuses on reducing error rates and increasing contig sizes, usually evaluated by N50 (at least half the assembled nucleotides are part of contigs of length N50 or longer) [16]. Strategies to improve quality

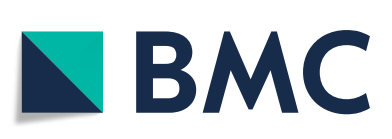

(c) The Author(s). 2019 Open Access This article is distributed under the terms of the Creative Commons Attribution 4.0 International License (http://creativecommons.org/licenses/by/4.0/), which permits unrestricted use, distribution, and reproduction in any medium, provided you give appropriate credit to the original author(s) and the source, provide a link to the Creative Commons license, and indicate if changes were made. The Creative Commons Public Domain Dedication waiver (http://creativecommons.org/publicdomain/zero/1.0/) applies to the data made available in this article, unless otherwise stated. 
include gene-boosted [17] and homology-guided assembly [18], which use existing information from related sequences to improve assembly results.

Despite advances in assembly software, assembling the massive amount of short read data necessary for de novo genome assembly is still a difficult technical task [19]. For eukaryotic genomes, de novo assembly typically requires high-performance computing resources with large memory and fast processors. Even with such extensive resources, it may take hours or days for completion of a single assembly attempt. If the resulting assembly is not satisfactory, parameter adjustments for subsequent runs and comparative evaluation of different draft assemblies are typically required. All of these challenges must ultimately be overcome to get a reliable whole-genome assembly.

However, whole-genome assembly is not necessarily the immediate, nor the only, goal of genome-wide NGS approaches. Because of the cost-effectiveness of NGS technologies, a research group may well choose genomewide NGS for a species even if they are interested in only a subset of the species' genes: for example, homologs of genes already identified in other species as being involved in a specific biochemical pathway or cellular structure. Alternatively, researchers can take advantage of the petabases of sequencing reads already present in the International Nucleotide Sequence Database Collaboration Sequence Read Archive (SRA) [20], which may include read deposits of interest for which no publicly available whole genome assemblies are available. Preassembly stages of massive read collections will also likely be a component of data release from large-scale sequencing projects such as the Earth BioGenome Project [21]. In these cases, it becomes expedient to restrict the assembly to the genic regions of interest; that is, instead of assembling the entire genome, assembling only the reads which correspond to annotated homologous genes of interest. By limiting the assembly to specific genomic regions, required resources and running time can be drastically reduced, and interpretation of output can be easily focused on the desired comparison of homologous regions. In pursuit of this goal, we have developed the program SRAssembler (Selective Recursive local Assembler).

SRAssembler uses a protein or DNA sequence from a related species as a query input to select and assemble NGS reads from a sequencing project in a different species or individual of interest (Fig. 1). Reads that are potentially homologous to the query sequence are assembled into contigs that serve as queries for the next recursive round of searching the reads, representing an "in silico" chromosome walking strategy as originally developed for mining the now outdated NCBI Trace Archive with the Tracembler program [22]. The user specifies success criteria that determine the break condition for the recursion, and at the last stage, the original query is aligned against the assembled contigs using spliced alignment software to identify potential gene structures.

Searching for matching reads based on the sequence of assembled contigs is a strategy also used by assembly gapfilling software such as GapFiller [23]. Indeed, SRAssembler could also be used for specific gap-filling simply by providing the sequences surrounding the gap as a target. What distinguishes SRAssembler is the use of local de novo assembly of matching reads rather than genomewide reference-based assembly, as well as the ability to use cDNA and protein probes for targeted assembly.

SRAssembler will also be useful to explore parameter spaces for whole genome assembly in a time-efficient manner. For a set of genes strongly expected to be present in a genome currently being sequenced, different assembly parameters can be explored and evaluated as to completeness of assembly of this diagnostic set of genes. The best parameter set can then be used for whole genome assembly. Similarly, completeness of assembly of such a gene set may also give insight into any additional sequencing needed for sufficient coverage of a novel genome of unknown size. If all the diagnostic genes can be locally assembled, then current coverage is likely adequate, whereas incomplete assembly may indicate the need for additional sequencing.

We illustrate the functionality of SRAssembler with examples using both simulated and genuine sequencing reads. We compare the efficacy of SRAssembler relative to whole-genome assembly and demonstrate that SRAssembler can not only assemble the likely orthologous gene, but can also distinguish and recover related paralogous genes using a single query. We show how the tool can be used to study gene body and promoter region variation using population genome data that are available merely as relatively low coverage, unassembled read deposits.

\section{Implementation}

SRAssembler is implemented as a $\mathrm{C}++$ program that relies on a number of freely available external programs for string matching, assembly, and spliced alignment. Default minimal prerequisites are Vmatch [24], SOAPdenovo2 [15], and GenomeThreader [25]. The program can be compiled with any standard $\mathrm{C}++$ compiler, or under openMPI for a multi-processor parallel computing platform[26]. Because SRAssembler is implemented within an object-oriented framework, advances in alignment and assembly software can be easily incorporated as new options within the workflow. Currently SRAssembler supports contig assembly with SOAPdenovo2 or ABySS, spliced alignment with GenomeThreader or GeneSeqer [27], and de novo gene finding with SNAP [28]. 


\section{In silico chromosome walking strategy}

The basic strategy implemented in SRAssembler is depicted in Fig. 1. Initially, NGS reads are aligned to a query sequence using the fast string matching program Vmatch. Query sequences can be either protein or DNA sequences provided in FASTA format. If the query sequence is a protein, the matching is to all possible translations of the reads (Vmatch option -dnavsprot).

Retrieved reads from the initial matching are assembled into contigs that become query sequences for subsequent rounds of in silico chromosome walking. Thus, in each round of the workflow, larger sets of presumed relevant read are retrieved from the input and assembled until one of alternative stopping criteria are met (see below), at which point the assembled contig(s) will contain the sought homologous gene or the program declares failure of the search within the given criteria. In cases when read coverage is expected to be low or when only short contigs were assembled in round 1 for later searches, SRAssembler can be run with the command-line '-a' flag to set a later round to begin assembling found reads into contigs. Until that round is completed, reads found by SRAssembler will be used directly as queries, allowing reads that could not be assembled into contigs a better chance of finding overlapping reads.

\section{Preprocessing reads}

Input read files can be in either FASTQ or FASTA format and single-end or paired-end. If a read library is pairedend, the reads must be in two sorted files rather than a single interleaved file. Although SRAssembler accepts single-end reads, paired-end reads typically provide better results because they allow reads not matching exons to be found more quickly. SRAssembler supports assembly from multiple read libraries simultaneously.

SRAssembler can take advantage of multiple processors to parallelize the search for new reads using the Message Passing Interface (MPI) protocol. To facilitate this, input reads data are split into several chunks. Each chunk is indexed by Vmatch, allowing very fast searching of the reads for matches to query contigs. These processed reads can be used again for subsequent SRAssembler runs.

\section{Read assembly}

At the end of each workflow round, after searching for new reads with Vmatch, SRAssembler assembles all the

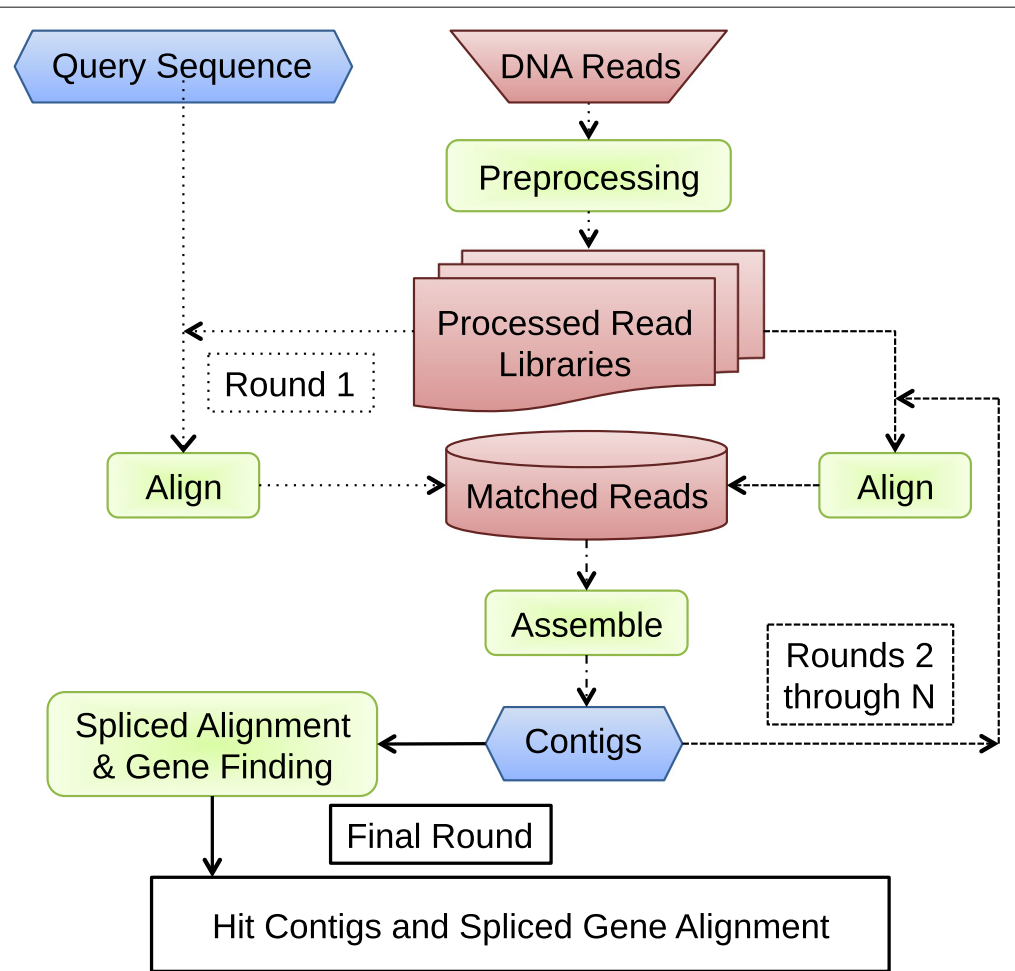

Fig. 1 SRAssembler workflow. The pipeline takes as input a query sequence and DNA sequencing read files, which are split into smaller files in a preprocessing step, allowing for parallelization of alignments. In the initial round, SRAssembler aligns the processed reads against the query (DNA or protein) sequence to identify reads that could potentially contribute to a homologous contig. Matched reads are then assembled into contigs, which are used as queries in the next round of searching the read libraries. The reads found in recursive rounds are reassembled, extending the edges of the contigs until no further matching reads are found or until user criteria for success are met (usually a finite number of rounds or complete coverage of the query). In the final round a spliced alignment program aligns the original query to the final contigs, predicting contigs that are good matches for the query and their homologous gene structure(s) 
reads it has found so far into new contigs. By default, SRAssembler invokes SOAPdenovo2 for the assembly step. The ABySS assembler may be used instead at the user's discretion. During the assembly step, the assembler is run multiple times with different $k$-mer values (the default setting uses $15,25,35$, and 45 ). The contigs of each assembly are compared to the query sequence using spliced alignment software (by default, GenomeThreader, with the option of GeneSeqer also available). The k-mer size that produces the greatest spliced alignment length is considered to be the best k-mer of that round, and the contigs produced by that $\mathrm{k}$-mer will become the query sequences for the next round. Before the contigs are used, very short contigs (by default shorter than $200 \mathrm{bp}$ ) are removed, and low complexity regions of the remaining contigs are masked with NCBI's DustMasker [29].

\section{Cleaning non-matching contigs and reads}

Periodically (every four rounds, by default), assembled contigs and found reads will be culled of non-matches, which can slow SRAssembler and impact the results. During these "cleaning rounds," assembled contigs are matched against the original protein or DNA query using Vmatch, and any contigs that do not have at least partial matches to the query are discarded. This can happen, for example, when the queries contain repetitive sequences that would match elsewhere in the genome apart from their occurrence in the gene of interest. After non-matching contigs have been removed, all of the reads that have been found so far are matched to the remaining contigs, and any reads that do not match (and therefore were not assembled into the matching contigs) are also discarded. SRAssembler will also perform cleaning at the end of a round that produces a number of contigs higher than a threshold (default 500 contigs). This is to prevent slowdown caused by the assembly of a highly duplicated region leading to an excess of contigs and reads unrelated to the query.

Cleaning non-matching contigs and reads improves the speed of SRAssembler and can prevent extraneous reads from interfering with the assembly of high quality contigs that match the query. However, the cleaning can also remove useful reads that would have been assembled into a matching contig in a later round, potentially preventing a hit contig from being extended. Adjusting the frequency of cleaning can be useful when attempting to extend a hit contig to more completely cover a region of interest, or when trying to find or complete additional paralogous sequences.

\section{Contig maximum length}

If an assembled contig is larger than the predefined maximum contig size (default 10,000 bp), that contig will be removed as a query for future rounds. The head and tail of these contigs are trimmed to make their size equal to the defined maximum contig size, and then are copied to the candidate-long-contig file. In the next round, any additional matching reads found by Vmatch using the contigs that did not exceed the maximum length are added to the pool of found reads. If long contigs assembled in this round match the candidate long contigs from the previous round (that is, the long contigs from the previous round are assembled again even with the addition of new reads), those candidate long contigs are moved to the permanent long contig file. The pool of matched reads is aligned to the permanent long contig file and any matching reads are removed from the pool in order to speed subsequent assemblies and prevent the long contigs from being extended any further. These long contigs are retained until recursion stopping criteria are met and are included in the final contig file.

\section{Stopping criteria}

The recursion is terminated as soon as one of the following criteria is met:

(1) Success - a hit contig is found. Here, a "hit contig" is defined as an assembled contig that satisfies the current user-set criteria for success: the contig length matches or exceeds the minimum specified value (default $200 \mathrm{bp}$ ); the spliced alignment similarity score of query versus contig is greater or equal to the threshold set (default 0.5 ); and the extent of the spliced alignment covers at least the specified minimum fraction of the query (default 0.8).

Alternatively, the assembly attempts will be stopped short of success in case of:

(2) No new reads can be found, meaning no contigs can be further extended.

(3) A specified maximum number of iterations is reached.

(4) All assembled contigs match or exceed the specified maximum length.

To determine if criterion 1 is met, the spliced alignment program is used to map the original query onto each round's assembled contigs. Criterion 1 can be ignored with the '-f' flag, forcing SRAssembler to complete the user-specified maximum number of rounds, potentially extending hit contigs beyond the boundaries of the homologous gene. Alternatively, the '-E' option can be used to compel SRAssembler to complete extra rounds after criterion 1 is met. These may be helpful if the user wants to extend assembled contigs into regions flanking the homologous coding region, but in some cases using the '-f' or '-E' options may lead to lower quality hit contigs, as extraneous reads can disrupt assembly in later rounds.

When SRAssembler cannot find a contig that meets the success criteria, it will run until one of criteria 2,3 , or 4 is met. This can occur because the source of the reads does not contain a homolog to the probe, because the success 
criteria are too stringent, or because of any of the various factors that affect read assembly.

\section{Final round}

After recursion is terminated, the contigs assembled in the final round that are longer than the minimum length are reported in the "all_contigs.fasta" file. Spliced alignment of the contigs assembled in the final round is used to create the "hit_contigs.fasta" that meet the user-specified criteria (mentioned above), and the spliced alignment file is available as "output.aln". If the probe contains a common protein domain, the "all_contigs.fasta" file may contain contigs that include that domain, but they will not be included in the "hit_contigs.fasta" file unless they meet the user-specifiable criteria for a good match. Optionally, an $a b$ initio gene finding program (currently SNAP is supported) will attempt to identify potential gene structure in the hit contigs and produce "output.ano", in addition to and independent of the spliced alignment.

\section{Results}

SRAssembler can be installed from our github repository https://github.com/BrendelGroup/SRAssembler, which also includes the manual and detailed instructions for installing prerequisite third-party software. Scripts to download the data used and to perform all of the analyses reported in this paper are included as Additional file 3. The simplest way to run SRAssembler on any single or multi-processor Linux system is via its containerized version as a Singularity image [30], which has all prerequisites bundled. The Singularity image of SRAssembler is available at Singularity Hub [31] at https://www.singularityhub.org/collections/1653. SRAssembler version 1.0.0 was used for the experiments in this manuscript.

\section{Assembly of homologous loci from simulated data}

The goal of the SRAssembler strategy is to construct local assemblies of NGS reads that encode putative homologs of a query protein or cDNA sequence. Because of our own expertise in plant genomics, our SRAssembler illustrations are mostly reported with plant genomic examples. The program is agnostic to the genomic sources of the reads, although parameter settings may have to be adjusted to fit characteristics of the genome.

To demonstrate the SRAssembler strategy, we used the rice protein sequence Os07g26940.1 as a query to try to assemble a contig containing the known homologous gene At1g01230 from simulated sequencing reads from Arabidopsis chromosome 1 . We simulated paired-end NGS sequencing using the SAMTools program wgsim [32]. The number of reads $\mathrm{N}$ was calculated as $\mathrm{N}=$ (length of chromosome $1 \times$ coverage) / (length of reads $\mathrm{x} 2$ ). Parameters were set as follows: base error rate 0.02, mutation 0 , and fraction of indels 0.10 . Read length was set to $70 \mathrm{bp}$, and insert size to $340 \mathrm{bp}$ with standard deviation $50 \mathrm{bp}$.

Figure 2 depicts the gene structure of At1g01230.1 and a spliced alignment produced by GenomeThreader of the Os07g26940.1 protein sequence against the final contig produced by an example SRAssembler run. At each of the portrayed rounds of recursion, the sequencing reads identified by SRAssembler as potentially part of a homologous locus are mapped (using Bowtie2 [33]) onto the final contig and visualized with the Integrative Genomics Viewer [34].

In the initial round, which uses the Os07g26940.1 protein sequence as the query to search the reads, SRAssembler finds reads that align to the exons. Because we simulated paired-end reads, both members of the pair are retained if either one of them aligns to the query. This leads to some low-depth coverage of the introns and untranslated regions (UTRs). During round 2, the contigs assembled from the reads found in round 1 are used to search for additional reads. By round 3 there is good read coverage of the full length of the target gene, and additional rounds find new reads at the contig borders that can extend its length. One observation from the results in Fig. 2 is that initial walking is very fast when connecting adjacent coding regions (i.e., exons), but extension of the contig boundaries is relatively slow. If a user's region of interest is flanking, rather than part of, the coding sequence (e.g., promoter elements), more rounds of recursion are necessary.

\section{Comparison of SRAssembler to whole genome assembly}

The ability to perform targeted local assembly is not useful if the results are low quality. To demonstrate the effectiveness of SRAssembler, we generated sets of simulated sequencing reads of Arabidopsis chromosome 1 with four depths of coverage ranging from $10 \mathrm{X}$ to $40 \mathrm{X}$. Twenty different random "seeds" (numbers used to create reproducible pseudo-random output) were used with wgsim at each coverage level, producing a total of 80 sets of reads. We used SOAPdenovo2, the default assembler used by the SRAssembler workflow, to attempt full assemblies of Arabidopsis chromosome 1 from each of the 80 read sets.

The rice-homologs track from PlantGDB AtGDB [35] indicates that 20 loci within the first million bases of Arabidopsis chromosome 1 have rice homologs that are over 100 amino acids in length. These 20 rice protein sequences (Table 1) were used as queries for SRAssembler against the simulated read sets. The contigs produced by SRAssembler from each run were aligned using BLAST+ [36] against the full gene sequence of their respective Arabidopsis orthologs. The assembly contigs produced by SOAPdenovo2 were also searched using BLAST + for the 20 aforementioned Arabidopsis genes. 


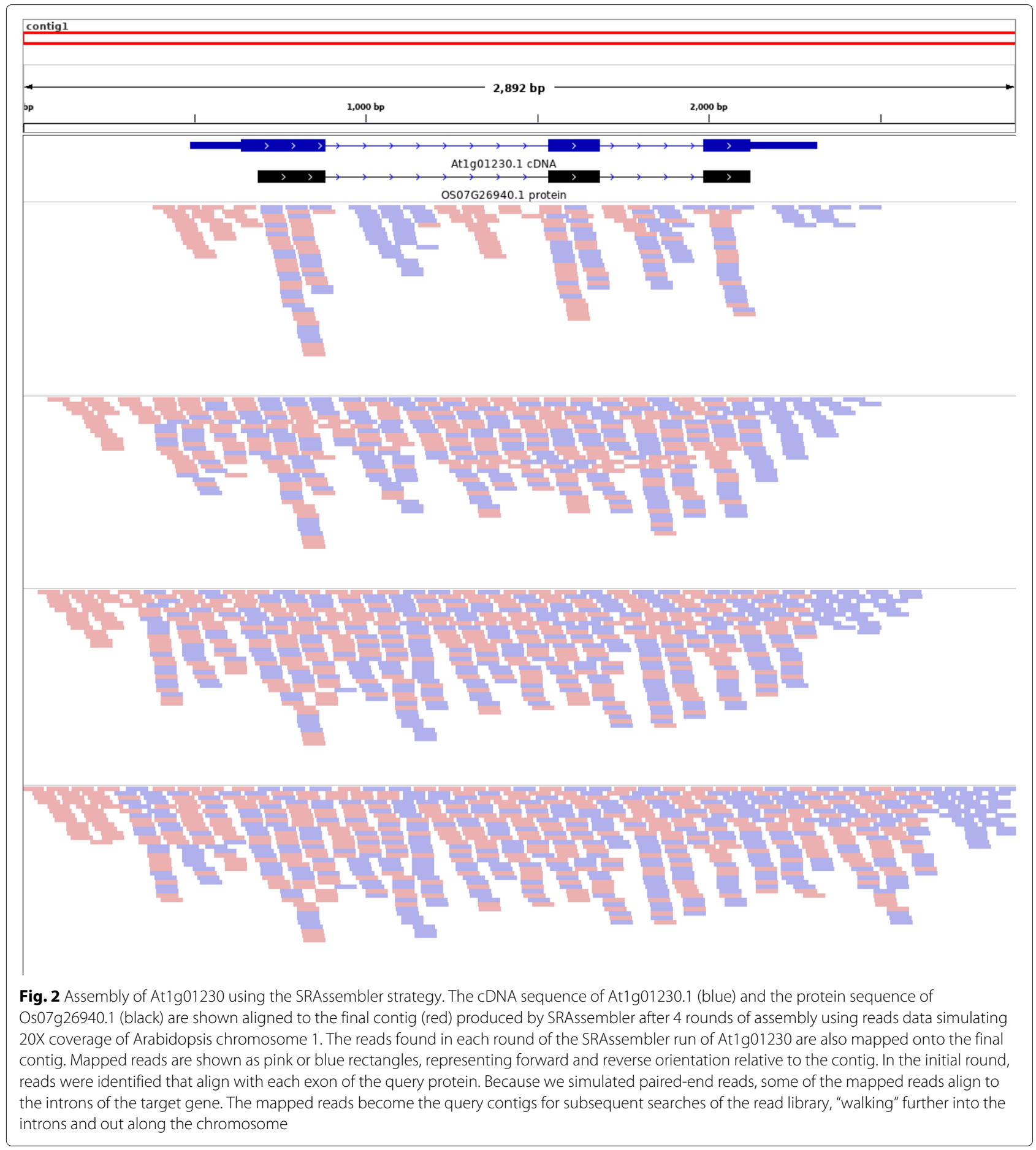

For both SRAssembler and SOAPdenovo2 the percent identity with the target reference sequence was over $98 \%$ in all cases, and over 99\% when read coverage was $20 \mathrm{X}$ or higher. Assembly completeness of the target gene tended to correlate with coverage depth, but there was surprising variance between read sets generated from different random seeds, especially at $10 \mathrm{X}$ and $20 \mathrm{X}$ coverage, and increasing coverage depth did not universally improve assembly (Additional file 1: Figure S1). Our metric of comparison was the percentage of the target gene sequence that aligned to the assembled contigs produced by SRAssembler and SOAPdenovo2 for each read set. In 1062 out of 1600 cases (66.4\%), SRAssembler and SOAPdenovo 2 performed equivalently. In 271 cases (16.9\%) SRAssembler produced a contig containing more of the target sequence, and in 267 cases (16.7\%) SOAPdenovo2 
Table 1 SRAssembly of 20 Arabidopsis genes

\begin{tabular}{|c|c|c|c|c|c|c|}
\hline Rice query & Arabidopsis ortholog & Locus start & Locus end & Contig start & Contig end & Identity \\
\hline Os03g02970.1 & At1g01040.2 & 23121 & 31227 & 23070 & 32269 & 99.99 \\
\hline Os05g36260.1 & At1g01050.1 & 31170 & 33171 & 30480 & 33907 & 99.97 \\
\hline Os04g02900.1 & At1g01090.1 & 47234 & 49304 & 47020 & 49718 & 100 \\
\hline Os07g26940.1 & At1g01230.1 & 97412 & 99240 & 96394 & 99684 & 99.85 \\
\hline Os08g06060.1 & At1g01560.2 & 202103 & 204440 & 201313 & 205079 & 100 \\
\hline Os07g26660.1 & At1g01620.1 & 225665 & 227543 & 224898 & 228253 & 100 \\
\hline Os02g44470.1 & At1g01750.2 & 275188 & 276310 & 274408 & 277381 & 100 \\
\hline Os06g03660.2 & At1g01820.1 & 296001 & 298334 & 295251 & 298848 & 100 \\
\hline Os09g34970.1 & At1g01910.1 & 313101 & 316090 & 312601 & 316984 & 100 \\
\hline Os06g04000.1 & At1g01940.1 & 322886 & 324917 & 321925 & 326047 & 99.98 \\
\hline Os06g04560.1 & At1g01950.3 & 325316 & 330619 & 325316 & 331264 & 100 \\
\hline Os01g08450.1 & At1g02130.1 & 399983 & 401919 & 399420 & 401973 & 100 \\
\hline Os12g18880.1 & At1g02140.1 & 403100 & 404456 & 402439 & 405644 & 99.94 \\
\hline Os01g18860.1 & At1g02500.1 & 518091 & 520495 & 517829 & 521327 & 100 \\
\hline Os03g19510.1 & At1g02560.1 & 537740 & 540127 & 537158 & 540531 & 100 \\
\hline Os03g21940.1 & At1g02780.1 & 607799 & 609534 & 607187 & 609669 & 100 \\
\hline Os03g22340.1 & At1g02830.1 & 625084 & 625608 & 624076 & 626451 & 100 \\
\hline Os05g05260.1 & At1g03190.1 & 775527 & 780062 & 774617 & 780567 & 99.87 \\
\hline Os04g52130.1 & At1g03475.1 & 868853 & 871308 & 868050 & 872005 & 99.62 \\
\hline Os10g35370.1 & At1g03630.1 & 907523 & 909650 & 907182 & 909855 & 99.93 \\
\hline
\end{tabular}

Twenty Arabidopsis genes chosen from the first million base pairs of chromosome 1 were assembled using rice protein queries. "Locus start" and "Locus end" are the coordinates of the loci of the Arabidopsis genes, and "Contig Start" and "Contig End" indicate where the SRAssembler result aligns to Arabidopsis chromosome 1 with BLASTN

was superior. SRAssembler was able to match or exceed the utility of a whole genome assembly in a total of 1333 cases $(83.3 \%)$.

\section{Assembly of homologous loci from real data}

In real-world experiments, NGS reads are typically not uniformly distributed over the genome sequence. The aforementioned 20 rice protein sequences were again used as queries for SRAssembler, this time to assemble sequencing reads from a Swedish accession of Arabidopsis [37].

We evaluated the performance of SRAssembler in this test by aligning the contigs it produced against the TAIR10 reference Arabidopsis genome using BLASTN. The results in Table 1 show that for each protein query, SRAssembler produced a contig that mapped to the chromosome region containing the expected Arabidopsis ortholog of the rice query. All 20 of these contigs share at least $99.5 \%$ identity with the Arabidopsis reference sequence and contain the entire homologous Arabidopsis locus.

As a test of application on a more complex genome, we also performed the reciprocal assemblies, using the proteins encoded by the Arabidopsis genes as probes for SRAssembler to build matching contigs from a set of reads from IRIS 313-11802, a cultivar of rice from the 3000 Rice Genomes Project [38]. Libraries ERR611677 to ERR611681 were used in this test, giving approximate 17X genome coverage. Contigs were built after several rounds of assembly and evaluated by the quality and extent of GenomeThreader spliced alignments of the corresponding known rice proteins. In 15 cases a contig covered over $90 \%$ of the rice protein (Table 2), clearly identifying a homologous gene, and in all cases at least part of a relevant gene was identified. Depending on the goals of the study, in practice a user can use any of the contigs as starting points for further assembly attempts, adding more reads data (if available) or extending contigs by further assembly rounds (SRAssembler will appropriately build on the previously generated results). A researcher may also consider designing primers for genomic PCR based on the assembly results in order to generate a more reliable sequence, targeted to the gene of interest.

\section{Assembly of paralogous loci}

SRAssembler often assembles multiple contigs, some of which are not ultimately of interest. These may contain distantly related genes, or just share a common domain with the query. Spliced alignment software such as GenomeThreader is used to identify the contigs labeled 
Table 2 SRAssembly of rice homologs

\begin{tabular}{|c|c|c|c|}
\hline $\begin{array}{l}\text { Arabidopsis } \\
\text { query }\end{array}$ & Rice homolog & $\begin{array}{l}\text { Spliced alignment } \\
\text { score }\end{array}$ & $\begin{array}{l}\text { Homolog } \\
\text { coverage }\end{array}$ \\
\hline At1g01040.1 & Os03g02970.1 & 0.997 & 1.000 \\
\hline At1g01050.1 & Os05g36260.1 & 0.714 & 1.000 \\
\hline At1g01090.1 & Os04g02900.1 & 0.997 & 1.000 \\
\hline At1g01230.1 & Os07g26940.1 & 1.000 & 1.000 \\
\hline At1g01560.2 & Os08g06060.1 & 0.967 & 1.000 \\
\hline At1g01620.1 & Os07g26660.1 & 0.597 & 0.992 \\
\hline At1g01750.1 & Os02g44470.1 & 0.780 & 1.000 \\
\hline At1g01820.1 & Os06g03660.2 & 0.676 & 1.000 \\
\hline At1g01910.1 & Os09g34970.1 & 0.889 & 0.843 \\
\hline At1g01940.1 & Os06g04000.1 & 0.992 & 1.000 \\
\hline At1g01950.3 & Os06g04560.1 & 0.492 & 0.800 \\
\hline At1g02130.1 & Os01g08450.1 & 0.786 & 0.738 \\
\hline At1g02140.1 & Os12g18880.1 & 0.778 & 0.981 \\
\hline At1g02500.1 & Os01g18860.1 & 1.000 & 1.000 \\
\hline At1g02560.1 & Os03g19510.1 & 0.820 & 0.908 \\
\hline At1g02780.1 & Os03g21940.1 & 0.656 & 0.480 \\
\hline At1g02830.1 & Os03g22340.1 & 0.696 & 0.841 \\
\hline At1g03190.1 & Os05g05260.1 & 0.996 & 0.955 \\
\hline At1g03475.1 & Os04g52130.1 & 1.000 & 0.913 \\
\hline At1g03630.1 & Os10g35370.1 & 0.900 & 0.981 \\
\hline
\end{tabular}

Twenty Arabidopsis proteins were used as queries into rice reads. The homologous rice proteins were spliced-aligned to the assembled contigs. The best result for each query is shown here

as "hits" against the query. In many cases, multiple hit contigs are the result of whole or partial genome duplication events. The ability to potentially identify and assemble paralogous (homologous due to duplication within a genome) as well as orthologous (homologous due to speciation) loci to genes of interest is an additional feature of the SRAssembler approach. This can be especially valuable in plants, which frequently undergo genetic duplication events.

The Arabidopsis Information Portal (Araport) [39] ThaleMine tool was used to identify genes paralogous to the 20 Arabidopsis loci from the previous section. Nineteen out of the 20 genes had at least one paralog identified in PANTHER version 11 [40]. Many of the contigs assembled by SRAssembler were identified by BLAST to correspond to one of these paralogs. Out of 295 Arabidopsis genes identified as homologous to the 20 rice queries, 79 (26.8\%) genes were completely assembled. In many cases the assembled contigs covered only part of a paralogous locus. Beyond the 79 complete genes, 19 additional gene bodies (the region from the start codon to the stop codon, but not including UTRs) were fully assembled, and a total of 141 gene bodies had at least $50 \%$ of their length covered by a contig. Table 3 summarizes these results, and Additional file 2: Table S1 contains details for each paralogous gene individually.

\section{Intra-species comparison of gene homologs}

Twenty representative cultivars from the 3000 Rice Genomes Project were selected to demonstrate the utility of SRAssembler for analyzing conservation of a gene within a species. The coding sequence of Os07g26940.1 was used as an example query. A homologous contig was successfully assembled from each of the cultivars. These contigs were aligned to the reference Os07g26940.1 gene sequence with MUSCLE [41] and show strong conservation in both exons and introns (alignment included as Additional file 4). Exon 1 has 99.5\% identical sites, intron 1 has $98.9 \%$ identical sites, exon 2 has $100 \%$ identical sites, intron 2 has $96.8 \%$ identical sites, and exon 3 has 99.3\% identical sites. The $5^{\prime}$-UTR has $92.1 \%$ identical sites, and the 3'-UTR has $99.7 \%$ identical sites. The $301 \mathrm{bp}$ region of the multiple sequence alignment upstream of

Table 3 Summary of assembly of paralogous Arabidopsis genes

\begin{tabular}{lllll}
\hline Arabidopsis & $\begin{array}{l}\text { Total } \\
\text { paralogs in } \\
\text { clade }\end{array}$ & $\begin{array}{l}\text { Complete } \\
\text { paralogs }\end{array}$ & $\begin{array}{l}\text { Complete } \\
\text { gene } \\
\text { bodies }\end{array}$ & $\begin{array}{l}\text { Gene bodies } \\
>50 \% \\
\text { assembled }\end{array}$ \\
\hline At1g01040.2 & 6 & $1(17 \%)$ & $1(17 \%)$ & $1(17 \%)$ \\
At1g01050.1 & 6 & $4(67 \%)$ & $4(67 \%)$ & $5(83 \%)$ \\
At1g01090.1 & 3 & $1(33 \%)$ & $1(33 \%)$ & $1(33 \%)$ \\
At1g01230.1 & 2 & $1(50 \%)$ & $2(100 \%)$ & $2(100 \%)$ \\
At1g01560.2 & 29 & $3(10 \%)$ & $5(17 \%)$ & $17(59 \%)$ \\
At1g01620.1 & 37 & $12(32 \%)$ & $14(38 \%)$ & $17(46 \%)$ \\
At1g01750.2 & 12 & $9(75 \%)$ & $10(83 \%)$ & $10(83 \%)$ \\
At1g01820.1 & 5 & $3(60 \%)$ & $3(60 \%)$ & $3(60 \%)$ \\
At1g01910.1 & 3 & $1(33 \%)$ & $1(33 \%)$ & $3(100 \%)$ \\
At1g01940.1 & 30 & $3(10 \%)$ & $6(20 \%)$ & $12(40 \%)$ \\
At1g01950.3 & 64 & $1(2 \%)$ & $1(2 \%)$ & $10(16 \%)$ \\
At1g02130.1 & 63 & $22(35 \%)$ & $30(48 \%)$ & $40(63 \%)$ \\
At1g02140.1 & 1 & $1(100 \%)$ & $1(100 \%)$ & $1(100 \%)$ \\
At1g02500.1 & 4 & $3(75 \%)$ & $4(100 \%)$ & $4(100 \%)$ \\
At1g02560.1 & 10 & $2(20 \%)$ & $3(30 \%)$ & $3(30 \%)$ \\
At1g02780.1 & 4 & $3(75 \%)$ & $3(75 \%)$ & $3(75 \%)$ \\
At1g02830.1 & 3 & $3(100 \%)$ & $3(100 \%)$ & $3(100 \%)$ \\
At1g03190.1 & 6 & $1(17 \%)$ & $1(17 \%)$ & $1(17 \%)$ \\
At1g03475.1 & 2 & $2(100 \%)$ & $2(100 \%)$ & $2(100 \%)$ \\
At1g03630.1 & 5 & $3(60 \%)$ & $3(60 \%)$ & $3(60 \%)$ \\
\hline Nin)
\end{tabular}

Nineteen out of the 20 Arabidopsis gene "targets" have at least one annotated paralog. SRAssembler was able to completely assemble at least one additional paralog for 13 of those targets. In many cases in which the complete paralog was not assembled, contigs still covered a significant fraction of the gene body (region from the start codon to the stop codon). If further investigation of a clade is desired, the final contigs could be used as starting queries for new SRAssembler runs 
the Os07g26940.1 start site is also highly conserved, with 90.4\% identical sites.

Further upstream of this point, nine of the cultivars diverge widely from the other eleven. Based on comparison to the rice reference Os-Nipponbare-ReferenceIRGSP-1.0 [42], it appears that these cultivars likely share an insertion of at least $400 \mathrm{bp}$. This is notable because this variance from the reference is not reported in the Rice SNP-seek database [43] for any of the nine divergent cultivars (and obviously could not be reported, as the database data are derived from read mapping to the reference genome, which would necessarily miss anything longer than within-read length insertions or deletions).

\section{Assembly from short reads in RNAseq data}

SRAssembler can also assemble contigs using sequencing reads from sources other than whole-genome sequencing experiments, such as RNAseq. RNAseq data from mouse skeletal muscle were used to assemble Myf6, a myogenic transcription factor gene [44]. Despite the reads being only 33 base pairs, SRAssembler was able to assemble a contig with $100 \%$ identity to the mRNA corresponding to the full length of the query protein.

\section{Running time}

Because SRAssembler directly assembles relatively short regions of interest, it takes far less time and computing power than a complete genome assembly. As a demonstration of the potential speed and resource use of SRAssembler, we used the rice protein Os07g26940.1 as a query for SRAssembler using 42 million Arabidopsis genomic read pairs from NCBI SRA ([20]) accession SRR519536. In these tests we ran the single-threaded SRAssembler with one processor and the openMPI-configured version of SRAssembler with $5,10,15$, and 20 processors, and compared the amount of time spent on preprocessing reads and recursively assembling contigs (Fig. 3). Preprocessing in SRAssembler splits read files into conveniently manageable sizes, converts the read format from FASTQ to FASTA to minimize the storage footprint, and indexes the reads for speedy searching. If SRAssembler is rerun using the same sequencing data, the preprocessing step

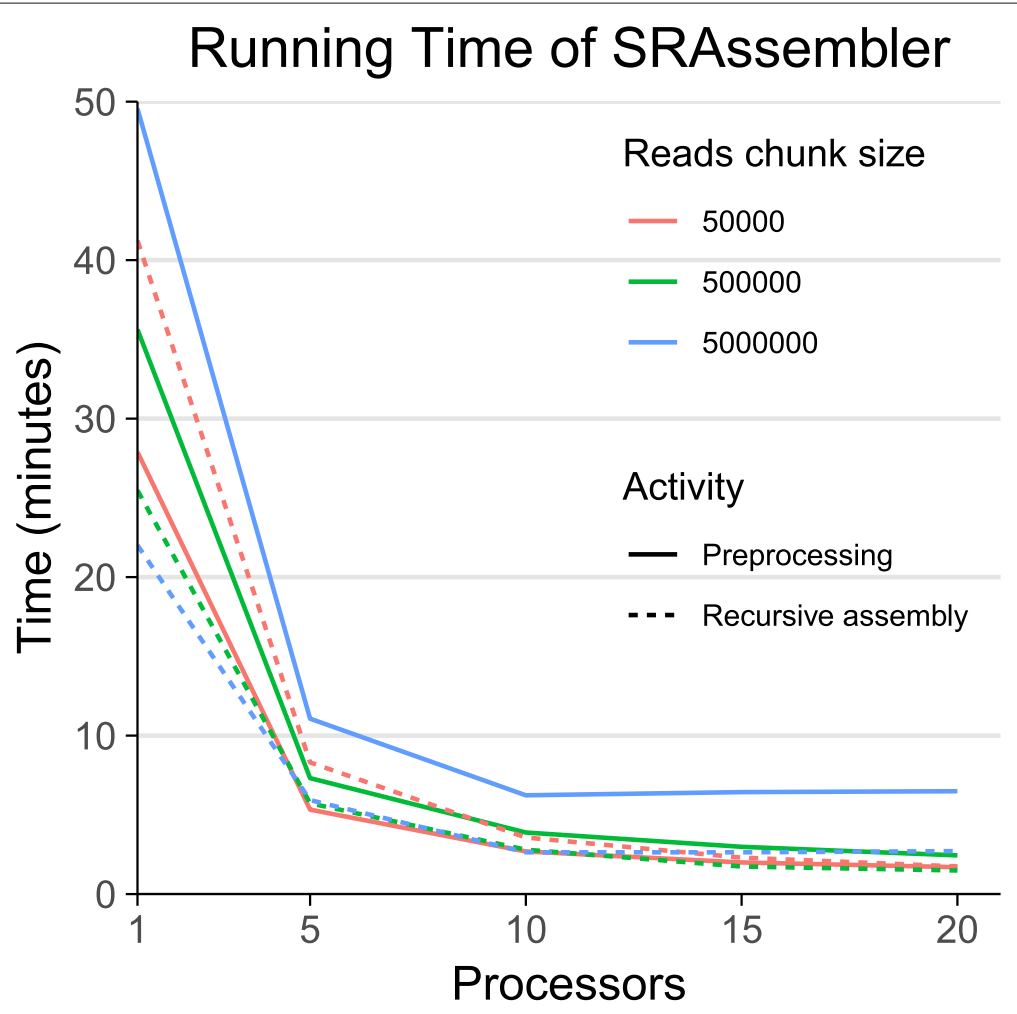

Fig. 3 Running time of SRAssembler. SRAssembler was run for five rounds using the Os07g26940.1 protein sequence as a query for 42 million Arabidopsis genomic read pairs. The effect on running time of pre-existing preprocessed read chunks, the size of the read chunks, and the number of processors assigned to SRAssembler were each tested and are shown as the mean of three technical replicates. As the number of processors assigned to SRAssembler rises from one to 17, completion time drops dramatically. Using larger read chunks increases the time required for preprocessing but can decrease the time of chromosome walking up to some point. The speed gains from increasing numbers of processors show diminishing returns, and vanish if the number of processors exceeds the number of read chunks 
can be skipped, improving running time. This is useful when assembling several homologs from the same sequencing data, or when experimenting with different run parameters.

The number of chunks the read files are split into can impact the speed of an SRAssembler run. Using fewer, larger files makes read processing take longer, but can make subsequent runs faster, at least when using one processor. The advantages of parallelization disappear when the number of chunk files exceeds the number of processors available.

When SRAssembler was run on a single core with the default split file size of 500,000 reads, the execution time of this test averaged $61 \mathrm{~min}$; this dropped to $4 \mathrm{~min}$ with 20 cores. A little more than half of this time was spent on the reads preprocessing stage. SRAssembler running time for a predetermined number of rounds is dependent on the number of processors and the size of the read libraries used, but is less predictable when stopping is based on completion of a contig that covers the query sequence.

\section{Discussion}

SRAssembler is not the first software to use a recursive search approach to analyze NGS reads that have not yet been assembled. Tracembler used recursive BLAST searches within the NCBI Trace Archive, but was not capable of searching user-provided read libraries, and used a less sophisticated approach to selecting and assembling reads [22]. The Mapsembler program is a tool targeting specific biological events such as SNPs, splicing events, or gene fusion [45]. Although Mapsembler also uses an iterative search algorithm as in Tracembler and SRAssembler, it is not designed to assemble homologous loci, does not use paired-end reads, and will not accept a protein query sequence. A tool called MITObim [46] uses a "baiting and iterative mapping" strategy similar to our "in silico chromosome walking" to assemble mitochondrial genomes, but it is not designed to assemble regions homologous to generic protein or DNA sequences.

\section{Conclusions}

SRAssembler offers a fast, efficient way to assemble whole-genome sequencing reads into contigs containing regions of interest, and we expect this approach to be useful to biology researchers for a variety of purposes. One obvious use case is a researcher who is interested in the sequence of a specific gene in an organism that does not have an assembled genome. This could be a particularly interesting individual or population from a species with an existing reference genome, or perhaps a member of a previously unsequenced species. Rather than spending time and computational resources on whole-genome assembly (a process which still intimidates many biologists), SRAssembler is intended to allow the researcher to assemble the sequence they care about and move forward with their research questions. Our testing suggests that the assembly of a target homologous gene has accuracy similar to full genome assemblies by modern software, while being much faster and requiring fewer resources.

The speed and computational resource advantages of SRAssembler over whole-genome assembly become even more apparent when trying to scale up an experiment. If a researcher is interested in a specific gene family, not within a single individual, but in each of 500 members of a population, computational resources and time are likely to be more prohibitive than the falling costs of NGS technology. This is especially true if the researcher is using pre-existing sequencing reads.

Whether or not an SRAssembler assembly attempt succeeds depends on the specifics of the application, including factors such as available read depth, intron content and length of the target gene, repetitive sequence content of the target region, and degree of similarity between the probe and target sequences. In favorable conditions, a quick success is likely. In difficult conditions, a variety of SRAssembler options allow flexible use of the program that can often still lead to success.

Collections like the human 1000 Genomes project [47] and the 3000 Rice Genomes Project mean that researchers can perform new bioinformatic experiments without needing to collect new data. Sequencing reads collections have already been used to create tools like the Rice SNPseek database, which mapped sequencing reads onto the rice Nipponbare reference genome to detect SNPs and other small variants in each of the sequenced cultivars [42]. However, as demonstrated in one of our experiments, this method is not sufficient to detect larger variants such as long indels or chromosome rearrangements. The SRAssembler strategy of recursively searching for reads that match the region of interest is not biased by an existing reference sequence, and can lead to discoveries that reference-mapping alone cannot.

Ongoing and future massive sequencing projects (ultimately, the Earth BioGenome Project [21]) will generate unprecedented opportunities for detailed comparative genomics studies. SRAssembler should be a useful tool to aid in the transformation of such sequence data into knowledge.

\section{Availability and requirements}

Project name: SRAssembler

Project home page: https:/github.com/BrendelGroup/ SRAssembler

Operating system: UNIX-like

Programming language: $\mathrm{C}++$

Other requirements: Singularity v2.4.2+, OR Vmatch v2.3.0, SOAPdenovo2 v2.04, ABySS v2.1.0, GeneSeqer, GenomeThreader v1.7.0+, and SNAP v2006-07-28 


\section{License: GNU GPL-3.0 \\ Any restrictions to use by non-academics: None}

\section{Additional files}

Additional file 1: Figure comparing assembly of target genes from simulated reads as a factor of read coverage depth. Twenty Arabidopsis genes were assembled from 20 different sets of simulated reads ("seeds"), at four different read coverage depths. Although the majority of gene/seed combinations showed improved assembly of the gene with increasing coverage depth, each gene had at least one seed that produced a worse result at higher coverage depth than it had at a lower depth (demonstrated by negative line slopes). (PDF $13 \mathrm{~kb}$ )

Additional file 2: Table of Arabidopsis orthologs to rice queries and their aligned contigs. The contigs from twenty SRAssembler runs using rice proteins against Arabidopsis genomic sequencing reads were aligned with blastn against the Arabidopsis reference genome. The Arabidopsis orthologs ("Atgene"), their locations("GeneStart" and "GeneStop"), and the beginning and end of their coding sequences ("ORFstart" and "ORFstop") were identified with Araport Thalemine. If a contig from the SRAssembler run using the homologous rice protein ("Osprobe") overlapped with an Arabidopsis gene location, this is noted in the "Contig" column, as is the percent identity with ("Identity") and location on ("ContigStart" and "ContigStop") the reference genome. The length of overlap between the contig and each gene locus ("ContigGeneOverlap") and coding region ("ContigORFOverlap") are noted, as are the fraction of the total gene and coding region lengths ("GeneCoverage" and "ORFCoverage"). (TSV 30 kb)

Additional file 3: Scripts and files for complete replication of results. Unpacking this tarball will create directories and scripts that, when executed, will download all necessary reads and perform all of the experiments described in this manuscript. The scripts will reproduce all of the tables and figures in this manuscript except for the workflow diagram. (TAR $340 \mathrm{~kb}$ )

Additional file 4: FASTA-format alignment of hit contigs from 20 rice cultivars. The Os07g26940.1 CDS sequence was used as the SRAssembler query against sequencing reads from 20 rice cultivars. The hit contigs were aligned with the reference genomic sequence for Os07g26940.1 using MUSCLE. (MUSCLE $154 \mathrm{~kb}$ )

\section{Abbreviations}

MPI: Message passing interface; NGS: Next-generation sequencing; SRA: Sequence read archive; UTR: Untranslated region

\section{Acknowledgments}

We would like to acknowledge Jacqueline Abdo and William Ehlhardt for their help debugging SRAssembler.

\section{Authors' contributions}

TWM designed and implemented parts of the software and wrote the manuscript. HC designed and implemented parts of the software and contributed to the manuscript. VB conceived the algorithm, provided ideas and suggestions at all stages, and contributed to code and manuscript writing. All authors have read and approved the final manuscript.

\section{Funding}

This work used the Extreme Science and Engineering Discovery Environment (XSEDE) Jetstream resource at Indiana University and the Texas Advanced Computing Center through allocation TG-BIO160012 (Computational Genomics) to V.B. XSEDE is supported by National Science Foundation grant number ACl-1548562. The funding bodies did not play any role in the design of this study, nor the collection, analysis, or interpretation of data, nor in writing this manuscript.

\section{Availability of data and materials}

The sequence for the TAIR10 Arabidopsis thaliana chromosome 1 reference sequence is available from The Arabidopsis Information Resource [48]. The Arabidopsis thaliana sequencing reads dataset analyzed during the current study are available in the European Bioinformatics Institute SRA database, accession SRR519536 [37]. The cultivars of rice used in the current study were: IRIS 313-11737, IRIS 313-10603, IRIS 313-10177, CX357, IRIS 313-11643, IRIS 313-11671, IRIS 313-11723, IRIS 313-11736, IRIS 313-11790, IRIS 313-11794, IRIS 313-11800, IRIS 313-11812, IRIS 313-11924, IRIS 313-15910, IRIS 313-8326, IRIS 313-8493, IRIS 313-8658, IRIS 313-8665, IRIS 313-8669, and IRIS 313-11802. Information on where to find their sequencing reads is available through the 3000 Rice Genomes Project [38]. The mouse sequencing reads dataset analyzed during the current study are available in the European Bioinformatics Institute SRA database, accessions SRR001361 and SRR001362 [44]. All other data generated or analyzed during this study are included in this published article and its supplementary information files.

\section{Ethics approval and consent to participate}

Not applicable.

\section{Consent for publication}

Not applicable.

\section{Competing interests}

The authors declare that they have no competing interests.

\section{Author details}

${ }^{1}$ Department of Biology, Indiana University, Bloomington 47405, Indiana USA.

2 Department of Oncology, St Jude Children's Research Hospital, Memphis 38105, Tennessee, USA. ${ }^{3}$ Department of Computer Science, Indiana University, Bloomington 47405, Indiana, USA.

Received: 19 October 2018 Accepted: 13 June 2019

Published online: 02 July 2019

\section{References}

1. Metzker ML. Sequencing technologies - the next generation. Nat Rev Genet. 2010;11(1):31-46. https://doi.org/10.1038/nrg2626.

2. Fröhlich $\mathrm{H}$, Balling $\mathrm{R}$, Beerenwinkel $\mathrm{N}$, Kohlbacher $\mathrm{O}$, Kumar S, Lengauer T, Maathuis MH, Moreau Y, Murphy SA, Przytycka TM, Rebhan M, Röst H, Schuppert A, Schwab M, Spang R, Stekhoven D, Sun J, Weber A, Ziemek D, Zupan B. From hype to reality: data science enabling personalized medicine. BMC Med. 2018;16(1):150. https://doi.org/10. 1186/s12916-018-1122-7.

3. Yan C, Lin P, Lyu T, Hu Z, Fan Z, Li X, Yao X, Li J, Yin H. Unraveling the roles of regulatory genes during domestication of cultivated camellia: Evidence and insights from comparative and evolutionary genomics. Genes. 2018;9(10):. https://doi.org/10.3390/genes9100488.

4. Korbel JO, Urban AE, Grubert F, Du J, Royce TE, Starr P, Zhong G, Emanuel BS, Weissman SM, Snyder M, Gerstein MB. Systematic prediction and validation of breakpoints associated with copy-number variants in the human genome. Proc Natl Acad Sci USA. 2007;104(24): 10110-5. https://doi.org/10.1073/pnas.0703834104.

5. Bentley DR, Balasubramanian S, Swerdlow HP, Smith GP, Milton J, Brown CG, Hall KP, Evers DJ, Barnes CL, Bignell HR, Boutell JM, Bryant J, Carter RJ, Keira Cheetham R, Cox AJ, Ellis DJ, Flatbush MR, Gormley NA, Humphray SJ, Irving $\sqcup$, Karbelashvili MS, Kirk SM, Li H, LiuX, Maisinger KS, Murray LJ, Obradovic B, Ost T, Parkinson ML, Pratt MR, Rasolonjatovo IMJ, Reed MT, Rigatti R, Rodighiero C, Ross MT, Sabot A, Sankar SV, Scally A, Schroth GP, Smith ME, Smith VP, Spiridou A, Torrance PE, Tzonev SS, Vermaas EH, Walter K, Wu X, Zhang L, Alam MD, Anastasi C, Aniebo IC, Bailey DMD, Bancarz IR, Banerjee S, Barbour SG, Baybayan PA, Benoit VA, Benson KF, Bevis C, Black PJ, Boodhun A, Brennan JS, Bridgham JA, Brown RC, Brown AA, Buermann DH, Bundu AA, Burrows JC, Carter NP, Castillo N, Chiara E Catenazzi M, Chang S, Neil Cooley R, Crake NR, Dada OO, Diakoumakos KD, Dominguez-Fernandez B, Earnshaw DJ, Egbujor UC, E more DW, Etchin SS, Ewan MR, Fedurco M, Fraser $\sqcup$, Fuentes Fajardo KV Scott Furey W, George D, Gietzen KJ, Goddard CP, Golda GS, Granieri PA, Green DE, Gustafson DL, Hansen NF, Harnish K, Haudenschild CD, Heyer NI, Hims MM, Ho JT, Horgan AM, Hoschler K, Hurwitz S, Ivanov DV, Johnson MQ, James T, Huw Jones TA, Kang G-D, Kerelska TH, Kersey AD, Khrebtukova I, Kindwall AP, Kingsbury Z, Kokko-Gonzales PI, Kumar A, Laurent MA, Lawley CT, Lee SE, LeeX, Liao AK, Loch JA, Lok M, Luo S, Mammen RM, Martin JW, McCauley PG, McNitt P, Mehta P, Moon KW, Mullens JW, Newington T, Ning Z, Ling Ng B, Novo SM, O'Neill MJ, Osborne MA, Osnowski A, Ostadan O, Paraschos LL, Pickering L, Pike AC, 
Pike AC, Chris Pinkard D, Pliskin DP, Podhasky J, Quijano VJ, Raczy C, Rae VH, Rawlings SR, Chiva Rodriguez A, Roe PM, Rogers J, Rogert Bacigalupo MC, Romanov N, Romieu A, Roth RK, Rourke NJ, Ruediger ST, Rusman E, Sanches-Kuiper RM, Schenker MR, Seoane JM, Shaw RJ, Shiver MK, Short SW, Sizto NL, Sluis JP, Smith MA, Ernest Sohna Sohna J, Spence EJ, Stevens K, Sutton N, Szajkowski L, Tregidgo CL, Turcatti G, VandeVondele S, Verhovsky Y, Virk SM, Wakelin S, Walcott GC, Wang J, Worsley GJ, Yan J, Yau L, Zuerlein M, Rogers J, Mullikin JC, Hurles ME, McCooke NJ, West JS, Oaks FL, Lundberg PL, Klenerman D, Durbin R, Smith AJ. Accurate whole human genome sequencing using reversible terminator chemistry. Nature. 2008;456(7218):53-59. https://doi.org/10. 1038/nature07517.

6. Kidd JM, Cooper GM, Donahue WF, Hayden HS, Sampas N, Graves T, Hansen N, Teague B, Alkan C, Antonacci F, Haugen E, Zerr T, Yamada NA, Tsang P, Newman TL, Tuzun E, Cheng Z, Ebling HM, Tusneem N, David R, Gillett W, Phelps KA, Weaver M, Saranga D, Brand A, Tao W, Gustafson E, McKernan K, Chen L, Malig M, Smith JD, Korn JM, McCarroll SA, Altshuler DA, Peiffer DA, Dorschner M, Stamatoyannopoulos J, Schwartz D, Nickerson DA, Mullikin JC, Wilson RK, Bruhn L, Olson MV, Kaul R, Smith DR, Eichler EE. Mapping and sequencing of structural variation from eight human genomes. Nature. 2008;453(7191):56-64. https://doi.org/10.1038/ nature06862.

7. Cokus SJ, Feng S, Zhang X, Chen Z, Merriman B, Haudenschild CD, Pradhan S, Nelson SF, Pellegrini M, Jacobsen SE. Shotgun bisulphite sequencing of the Arabidopsis genome reveals DNA methylation patterning. Nature. 2008;452(7184):215-9. https://doi.org/10.1038/ nature06745.

8. Lister R, O'Malley RC, Tonti-Filippini J, Gregory BD, Berry CC, Millar AH, Ecker JR. Highly Integrated Single-Base Resolution Maps of the Epigenome in Arabidopsis. Cell. 2008;133(3):523-36. https://doi.org/10. 1016/j.cell.2008.03.029.

9. Mikkelsen TS, Ku M, Jaffe DB, Issac B, Lieberman E, Giannoukos G, Alvarez P, Brockman W, Kim T-K, Koche RP, Lee W, Mendenhall E, O'Donovan A, Presser A, Russ C, Xie X, Meissner A, Wernig M, Jaenisch R, Nusbaum C, Lander ES, Bernstein BE. Genome-wide maps of chromatin state in pluripotent and lineage-committed cells. Nature. 2007;448(7153): 553-60. https://doi.org/10.1038/nature06008.

10. Wederell ED, Bilenky M, Cullum R, Thiessen N, Dagpinar M, Delaney A, Varhol R, Zhao Y, Zeng T, Bernier B, Ingham M, Hirst M, Robertson G, Marra MA, Jones S, Hoodless PA. Global analysis of in vivo Foxa2-binding sites in mouse adult liver using massively parallel sequencing. Nucleic Acids Res. 2008;36(14):4549-64. https://doi.org/10.1093/nar/gkn382.

11. Pevzner PA, Tang H. Fragment assembly with double-barreled data. Bioinformatics. 2001;17(Suppl 1):225-33.

12. Zerbino DR, Birney E. Velvet: Algorithms for de novo short read assembly using de Bruijn graphs. Genome Res. 2008;18(5):821-9. https://doi.org/10. 1101/gr.074492.107.0209100.

13. Simpson JT, Wong K, Jackman SD, Schein JE, Jones SJM, Birol I. ABySS: A parallel assembler for short read sequence data. Genome Res. 2009;19(6): 1117-23. https://doi.org/10.1101/gr.089532.108.

14. Butler J, MacCallum I, Kleber M, Shlyakhter IA, Belmonte MK, Lander ES, Nusbaum C, Jaffe DB. ALLPATHS: de novo assembly of whole-genome shotgun microreads. Genome Res. 2008;18(5):810-20. https://doi.org/10. 1101/gr.7337908.

15. Luo R, Liu B, Xie Y, Li Z, Huang W, Yuan J, He G, Chen Y, Pan Q, Liu Y, Tang J, Wu G, Zhang H, Shi Y, Liu Y, Yu C, Wang B, Lu Y, Han C, Cheung DW, Yiu S-M, Peng S, Xiaoqian Z, Liu G, Liao X, Li Y, Yang H, Wang J, Lam T-W, Wang J. SOAPdenovo2: an empirically improved memory-efficient short-read de novo assembler. GigaScience. 2012; (1): 18. https://doi.org/10.1186/2047-217X-1-18.

16. Narzisi G, Mishra B. Comparing de novo genome assembly: the long and short of it. PLOS ONE. 2011;6(4):19175. https://doi.org/10.1371/journal. pone.0019175.

17. Salzberg SL, Sommer DD, Puiu D, Lee VT. Gene-Boosted Assembly of a Novel Bacterial Genome from Very Short Reads. PLoS Comput Biol. 2008;4(9):1000186. https://doi.org/10.1371/journal.pcbi.1000186.

18. Klein JD, Ossowski S, Schneeberger K, Weigel D, Huson DH. Locas - A low coverage assembly tool for resequencing projects. PLOS ONE. 2011;6(8):23455. https://doi.org/10.1371/journal.pone.0023455.

19. Richards S. Full disclosure: Genome assembly is still hard. PLoS Biol. 2018;16(4):1-5. https://doi.org/10.1371/journal.pbio.2005894.
20. Kodama $Y$, Shumway M, Leinonen $R$. The sequence read archive: explosive growth of sequencing data. Nucleic Acids Res. 2012;40(Database issue):54-56. https://doi.org/10.1093/nar/gkr854. 22009675[pmid].

21. Lewin HA, Robinson GE, Kress WJ, Baker WJ, Coddington J, Crandall KA, Durbin R, Edwards SV, Forest F, Gilbert MTP, Goldstein MM, Grigoriev IV Hackett KJ, Haussler D, Jarvis ED, Johnson WE, Patrinos A, Richards S, Castilla-Rubio JC, van Sluys M-A, Soltis PS, Xu X, Yang H, Zhang G. Earth biogenome project: Sequencing life for the future of life. Proc Natl Acad Sci USA. 2018;115(17):4325-33. https://doi.org/10.1073/pnas.1720115115.

22. Dong Q, Wilkerson MD, Brendel V. Tracembler-software for in silico chromosome walking in unassembled genomes. BMC Bioinformatics. 2007;8(1):151. https://doi.org/10.1186/1471-2105-8-151.

23. Nadalin F, Vezzi F, Policriti A. Gapfiller: a de novo assembly approach to fill the gap within paired reads. BMC Bioinformatics. 2012;13(14):8. https:// doi.org/10.1186/1471-2105-13-S14-S8.

24. Abouelhoda MI, Kurtz S, Ohlebusch E. Replacing suffix trees with enhanced suffix arrays. J Discret Algoritm. 2004;2(1 SPEC. ISS.):53-86. https://doi.org/10.1016/S1570-8667(03)00065-0.

25. Gremme G, Brendel V, Sparks ME, Kurtz S. Engineering a software tool for gene structure prediction in higher organisms. Inf Softw Technol. 2005;47(15):965-78. https://doi.org/10.1016/j.infsof.2005.09.005.

26. Graham RL, Woodall TS, Squyres JM. Open MPI: A Flexible High Performance MPI. In: Wyrzykowski R, Dongarra J, Meyer N, Waśniewski J, editors. Parallel Processing and Applied Mathematics. Berlin: Springer; 2006. p. 228-39.

27. Brendel V, Xing L, Zhu W. Gene structure prediction from consensus spliced alignment of multiple ESTs matching the same genomic locus. Bioinformatics. 2004;20(7):1157-69. https://doi.org/10.1093/ bioinformatics/bth058.

28. Korf I. Gene finding in novel genomes. BMC Bioinformatics. 2004;5(1):59. https://doi.org/10.1186/1471-2105-5-59.

29. Morgulis A, Gertz EM, Schaffer AA, Agarwala R. A fast and symmetric dust implementation to mask low-complexity dna sequences. J Comput Biol J Comput Mol Cell Biol. 2006;13(5):1028-40. https://doi.org/10.1089/ cmb.2006.13.1028.

30. Kurtzer GM, Sochat $V$, Bauer MW. Singularity: Scientific containers for mobility of compute. PLoS ONE. 2017;12(5):1-20. https://doi.org/10.1371/ journal.pone.0177459.

31. Sochat W, Prybol CJ, Kurtzer GM. Enhancing reproducibility in scientific computing: Metrics and registry for singularity containers. PLOS ONE. 2017;12(11):0188511. https://doi.org/10.1371/journal.pone.0188511.

32. Li H, Handsaker B, Wysoker A, Fennell T, Ruan J, Homer N, Marth G, Abecasis G, Durbin R, Data GP, Sam T, Subgroup GPDP. The Sequence Alignment / Map format and SAMtools. Bioinformatics. 2009;25(16): 2078-9. https://doi.org/10.1093/bioinformatics/btp352.

33. Langmead B, Salzberg SL. Fast gapped-read alignment with Bowtie 2 Nat Methods. 2012;9(4):357-9. https://doi.org/10.1038/nmeth.1923.

34. Robinson JT, Thorvaldsdóttir H, Winckler W, Guttman M, Lander ES, Getz G, Mesirov JP. Integrative genomics viewer. Nat Biotechnol. 2011;29(1):24-26. https://doi.org/10.1038/nbt.1754.

35. Duvick J, Fu A, Muppirala U, Sabharwal M, Wilkerson MD, Lawrence CJ, Lushbough C, Brendel V. PlantGDB: A resource for comparative plant genomics. Nucleic Acids Res. 2008;36(SUPPL. 1):959-65. https://doi.org/ 10.1093/nar/gkm 1041.

36. Camacho C, Coulouris G, Avagyan V, Ma N, Papadopoulos J, Bealer K, Madden TL. BLAST+: Architecture and applications. BMC Bioinformatics. 2009;10:1-9. https://doi.org/10.1186/1471-2105-10-421.

37. Long $Q$, Rabanal FA, Meng D, Huber CD, Farlow A, Platzer A, Zhang $Q$, Vilhjálmsson BJ, Korte A, Nizhynska V, Voronin V, Korte P, Sedman L, Mandáková T, Lysak MA, Seren Ü, Hellmann I, Nordborg M. Massive genomic variation and strong selection in Arabidopsis thaliana lines from Sweden. Nat Genet. 2013;45(8):884-90. https://doi.org/10.1038/ng.2678.

38. Li J-Y, Wang J, Zeigler RS. The 3,000 rice genomes project: new opportunities and challenges for future rice research. GigaScience. 2014;3(1):2047-1738. https://doi.org/10.1186/2047-217X-3-8. http://arxiv. org/abs//oup/backfile/content_public/journal/gigascience/3/1/10. 1186_2047-217x-3-8/8/13742_2014_article_41.pdf.

39. Krishnakumar $\mathrm{V}$, Hanlon MR, Contrino S, Ferlanti ES, Karamycheva S, Kim M, Rosen BD, Cheng CY, Moreira W, Mock SA, Stubbs J, Sullivan JM, 
Krampis K, Miller JR, Micklem G, Vaughn M, Town CD. Araport: The Arabidopsis Information Portal. Nucleic Acids Res. 2015;43(D1):1003-9. https://doi.org/10.1093/nar/gku1200.

40. Mi H, Huang X, Muruganujan A, Tang H, Mills C, Kang D, Thomas PD. PANTHER version 11: Expanded annotation data from Gene Ontology and Reactome pathways, and data analysis tool enhancements. Nucleic Acids Res. 2017;45(D1):183-9. https://doi.org/10.1093/nar/gkw1138.

41. Edgar RC. MUSCLE: multiple sequence alignment with high accuracy and high throughput. Nucleic Acids Res. 2004;32(5):1792-7. https://doi.org/ 10.1093/nar/gkh340.

42. Kawahara $Y$, de la Bastide M, Hamilton JP, Kanamori H, McCombie WR, Ouyang S, Schwartz DC, Tanaka T, Wu J, Zhou S, Childs KL, Davidson RM, Lin H, Quesada-Ocampo L, Vaillancourt B, Sakai H, Lee SS, Kim J, Numa H, Itoh T, Buell CR, Matsumoto T. Improvement of the Oryza sativa Nipponbare reference genome using next generation sequence and optical map data. Rice. 2013;6(1):1-10. https://doi.org/10.1186/19398433-6-1.

43. Mansueto L, Fuentes RR, Borja FN, Detras J, Abrio-Santos JM, Chebotarov D, Sanciangco M, Palis K, Copetti D, Poliakov A, Dubchak I, Solovyev V, Wing RA, Hamilton RS, Mauleon R, McNally KL, Alexandrov N. Rice SNP-seek database update: New SNPs, indels, and queries. Nucleic Acids Res. 2017;45(D1):1075-81. https://doi.org/10.1093/nar/gkw1135.

44. Mortazavi A, Williams BA, McCue K, Schaeffer L, Wold B. Mapping and quantifying mammalian transcriptomes by RNA-Seq. Nat Methods. 2008;5(7):621-8. https://doi.org/10.1038/nmeth.1226.

45. Peterlongo $P$, Chikhi R. Mapsembler, targeted and micro assembly of large NGS datasets on a desktop computer. BMC Bioinformatics. 2012;13(1):48. https://doi.org/10.1186/1471-2105-13-48.

46. Hahn C, Bachmann L, Chevreux B. Reconstructing mitochondrial genomes directly from genomic next-generation sequencing reads - A baiting and iterative mapping approach. Nucleic Acids Res. 2013;41(13). https://doi.org/10.1093/nar/gkt371.

47. Clarke L, Zheng-Bradley X, Smith R, Kulesha E, Xiao C, Toneva I, Vaughan $B$, Preuss D, Leinonen R, Shumway M, Sherry S, Flicek P. The 1000 Genomes Project: data management and community access. Nat Methods. 2012;9(5):459-62. https://doi.org/10.1038/nmeth.1974.

48. Lamesch P, Berardini TZ, Li D, Swarbreck D, Wilks C, Sasidharan R, Muller R, Dreher K, Alexander DL, Garcia-Hernandez M, Karthikeyan AS, Lee $\mathrm{CH}$, Nelson WD, Ploetz L, Singh S, Wensel A, Huala E. The Arabidopsis Information Resource (TAIR): Improved gene annotation and new tools. Nucleic Acids Res. 2012;40(D1):1202-10. https://doi.org/10. 1093/nar/gkr1090.

\section{Publisher's Note}

Springer Nature remains neutral with regard to jurisdictional claims in published maps and institutional affiliations.

Ready to submit your research? Choose BMC and benefit from:

- fast, convenient online submission

- thorough peer review by experienced researchers in your field

- rapid publication on acceptance

- support for research data, including large and complex data types

- gold Open Access which fosters wider collaboration and increased citations

- maximum visibility for your research: over $100 \mathrm{M}$ website views per year

At BMC, research is always in progress.

Learn more biomedcentral.com/submissions 